\title{
The effect of workload on nurses' quality of life with moderating perceived social support during the COVID-19 pandemic
}

\author{
Hossein Ebrahimi $^{\mathrm{a}}$, Ezzat Jafarjalal ${ }^{\mathrm{b}}$, Asghar Lotfolahzadeh ${ }^{\mathrm{c}}$ \\ and Seyedeh Melika Kharghani Moghadam ${ }^{\mathrm{d}, *}$ \\ ${ }^{a}$ Department of Occupational Health, School of Public Health, Iran University of Medical Sciences, Tehran, \\ Iran \\ ${ }^{\mathrm{b}}$ Department of Nursing Management, School of Nursing and Midwifery, Iran University of Medical Sciences, \\ Tehran, Iran \\ ${ }^{\mathrm{c}}$ Vice-Dean for Health, Ardabil University of Medical Sciences, Ardabil, Iran \\ ${ }^{\mathrm{d}}$ Department of Health Education and Health Promotion, School of Public Health, Iran University of Medical \\ Sciences, Tehran, Iran
}

Received 14 April 2021

Accepted 29 June 2021

\begin{abstract}
.
BACKGROUND: Due to the COVID-19 pandemic in Iran, the number of patients admitted to hospitals and the workload of nurses has increased. High workload can reduce efficiency and quality of life. In the meantime the percived social support may had a moderating role.

OBJECTIVE: The aim of the study was to investigate the effect of workload and perceived social support on quality of life in nurses who work in COVID-19 inpatient wards.

METHODS: This was a cross-sectional descriptive-analytic study. 336 nurses who worked in inpatient wards with COVID19 patients were randomly selected and studied. NASA-TLX Workload Questionnaire, WHO Quality of Life Questionnaire and Multidimensional Social Support Perception Scale were used to data collection. Structural equation modelling in PLS software was used to modelling.

RESULTS: The results showed that the average score of workload, perceived social support and quality of life were $80.87 \pm 20.17,56.23 \pm 11.46$ and $55.87 \pm 13.74$, respectively. A significant inverse relationship was observed between workload and quality of life $(P<0.05)$. Also, perceived social support had a moderator effect on relationship between workload and quality of life $(P<0.05)$.

CONCLUSIONS: High workload can upset the balance between work and life that leads to decrease the quality of life. Perceived social support as a moderator can reduce the negative impact of workload on quality of life. In the COVID-19 pandemic where nurses' workload have increased, by increasing the perceived social support can manage the negative effect of workload.
\end{abstract}

Keywords: Workload, quality of life, social support, nurses

*Address for correspondence: Seyedeh Melika Kharghani Moghadam, Department of Health Education and Health Promotion, School of Public Health, Iran University of Medical Sciences, Shahid Hemmat Highway, Tehran, 1449614535, Iran. Tel.: 09886704749; E-mail: m_kharghani@yahoo.com.

\section{Introduction}

The spread of a new and unknown viral infectious disease was reported at the end of December 2019 in Wuhan, China [1]. The cause of this disease was a 
new and genetically modified viruses namely SARS$\mathrm{CoV}-2$, which belongs to the family of Coronaviruses [2]. Therefore, the disease caused by this virus was named COVID-19 by the World Health Organization (WHO) $[3,4]$. The spread of COVID-19 is unique because of its transmission speed, which has led to create an emergency situation in the health of people in the community around the world in less than a few months [5]. In Iran, the COVID-19 pandemic has led to an increase in the number of cases of diseases and hospitalization. Consequently, an increase in hospitalization can increase the workload of nurses.

Workload is defined as "the amount of total work done by an individual or a team of individuals at a specified time interval" [6]. In addition to physical duties, workload is also due to the cognitive duties of nurses [7,8]. The nursing profession is considered as one of the most stressful professions. Heavy workload threatens the physical safety of nurses, and leads to reduce job satisfaction and increase occupational burnout [9]. Workload leads to reduce the quality of life in nurses $[10,11]$.

Quality of life is the general well-being of individuals and societies, outlining negative and positive features of life. It consists of the expectations of an individual or society for a good life. In the last decade, health-related quality of life assessment has been used extensively in medical research [12]. Quality of life is a broad multidimensional and mental concept that includes dimensions such as physical health, mental health, economic conditions, personal beliefs and interaction with the environment [13]. Nowadays, one of the major concerns of health professionals is quality of life and is used as an indicator to assess health status in health research [14]. Healthrelated quality of life is a subset of quality of life and goes back to people's experience of their overall health. Health-related quality is an individual's or a group's perceived physical and mental health over time. It can also be rooted in mental health well-being, functional status, or self-perceived health [15]. The perceived social support is one of the factors affecting the quality of life [16]. Social support is defined as "The amount of love, companionship, care, respect, attention, and assistance received by the individual by individuals or other groups such as family members, friends, and other important people in life." In other words, it can be defined as "helping individuals and groups within society that a barrier against adverse events and living conditions is provided by it and can be considered as a positive source for improving the quality of life" [15]. Social support means having friends and other people, including family, to turn to in times of need or crisis to give you a broader focus and positive self-image. It enhances quality of life and provides a buffer against adverse life events [15]. Perceived social support refers to how individuals perceive friends, family members and others as sources available to provide material, psychological and overall support during times of need [17]. A significant increase is observed in the number of hospitalized patients due to the Covid-19 epidemic in Iran. Increasing the number of patients admitted to hospitals can lead to increase the workload and consequently reduce the quality of life of nurses. In the meantime, perceived social support can act as a moderator and reduces the effect of workload on quality of life. So, the present study was carried out aimed to model the effect of workload on nurses' quality of life by the moderating role of social support during the COVID-19 pandemic.

\section{Conceptual model}

Workload is one of the effective factors on employees within organizations that have endangered the health of many people [17]. According to the results of studies, there is a direct relationship between workload and individual performance and is one of the components affecting health, safety and comfort [18]. According to the results, there is an inverse relationship between nurses 'workload and quality of life and workload is considered as one of the factors which reduce the quality of life of nurses [19-121]. According to the evidence obtained from various studies, social support plays an important role in people's health and has a significant effect on the quality of life by reducing the negative effects of many stresses that are obtained from the environment and society [17]. So, the conceptual model of Fig. 1 was proposed to investigate the effect of workload on quality of life by moderating effect of perceived social support.

There are 3 hypotheses based on the proposed conceptual model, which are:

Hypothesis 1: Workload has a significant effect on the quality of life.

Hypothesis 2: Perceived social support has a significant effect on the quality of life.

Hypothesis 3: Perceived social support as a moderator changes the effect of workload on quality of life. 


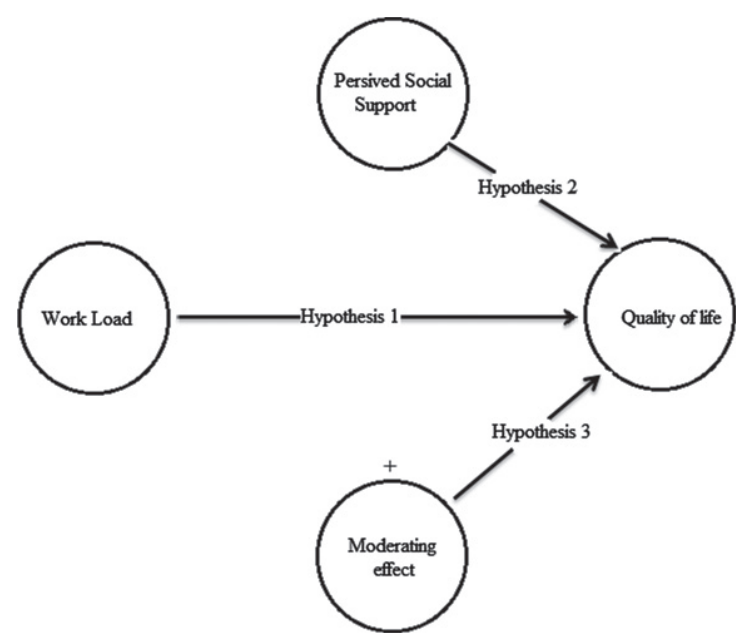

Fig. 1. The conceptual proposed model.

\section{Materials and methods}

\subsection{Target community}

This was a cross-sectional research conducted among the nurses of 5 hospitals affiliated to Iran University of Medical Sciences where COVID-19 patients were hospitalized. The study carried out between November and December, 2020. Inclusion criteria included being a nurse, working in these wards and lack of serious physical and mental health problems. Incompleteness of the completed questionnaire was also considered as an exclusion criterion. At least 200 samples are required based on the statistical method used (confirmatory factor analysis) [22]. Based on the study criteria, 336 subjects were randomly selected from studied hospitals.

\subsection{Data collection tools}

Three questionnaires of WHO quality of life consisting demographic data, NASA Task Load Index (NASA-TLX) and Multidimensional Scale of Perceived Social Support were used to collect information in the present study.

The World Health Organization quality of Life questionnaire is a 26-item questionnaire that measures the overall quality of life of individual. This scale was developed in 1996 by a group of experts from the World Health Organization by moderating effect of the items of the 100-question form of this questionnaire. This questionnaire measures subscales of physical health, mental health, social relationships, and environmental health with 24 questions [23]. Each domain has 7, 6, 3 and 8 questions, respectively. The first two questions do not belong to any of the areas and assess the state of health and quality of life in general. [24]. The questions have 5-Point Likert Scale which get a score of 1 to 5 , respectively. Thus the range of scores obtained for dimensions of physical health, mental health, social relationships, and environmental health were equal to 7-35, 6-30, 3-15 and $8-40$, respectively. Also, the range of obtained scores for quality of life was equal to $2-10$. In the next step, in order to equalize the range of scores for all dimensions, the following equation was used to convert the scores to a standard score between 0 and 100 .

$$
\begin{aligned}
& \text { Final } \\
& \text { score }
\end{aligned}=\frac{\left\{\begin{array}{c}
\text { Score obtained on a subscale- } \\
\text { The lowest possible subscale } \\
\text { score }
\end{array}\right\}}{\left\{\begin{array}{c}
\text { The difference between the } \\
\text { highest and lowest possible } \\
\text { scores of the subscale }
\end{array}\right\}} \times 100
$$

One of the most widely used tools for the mental workload assessment is the NASA-TLX Task Load Index that Hart and Steveland first used in 1988 for assessment of the mental workload of pilots [6]. Task Load Index composed by 6 subscales: Mental Demand, Physical Demand, Temporal Demand, Performance, Frustration and Effort. Each subscale includes one question. Hundred-step bipolar scales (0-100) are used to obtain ratings on these dimensions. [6].

The Multidimensional Scale of Perceived Social Support was developed by Zimet et al. in 1988 to assess perceived social support from family members, friends, and other important people in people's life [25]. The questionnaire consists of 12 questions and assesses perceived social support in three fields: family, friends and other important people. Each dimension has 4 questions with a Likert scale of 5 . The range of scores earned for each dimension and whole scale are between 4-20 and 12-60, respectively. A score between 12 and 20 indicates a low level, 20 to 40 a moderate level and above 40 a high level of perceived social support [25].

\subsection{Method}

The selected standard questionnaires were converted to electronic format in carry out the present study. The link of the designed electronic questionnaire was provided to the nurses through the 
Table 1

Mean scores of workload

\begin{tabular}{lcc}
\hline Work load subscales & Mean & SD \\
\hline Mental demand & 79.55 & 23.17 \\
Physical demand & 80.06 & 22.16 \\
Temporal demand & 87.24 & 18.12 \\
Performance & 74.45 & 19.34 \\
Effort & 79.28 & 20.83 \\
Frustration & 84.65 & 17.42 \\
Total score & 80.87 & 20.17 \\
\hline
\end{tabular}

Table 2

Mean score of perceived social support

\begin{tabular}{lcc}
\hline Perceived social support & Mean & SD \\
\hline Family support & 19.24 & 4.34 \\
Friends support & 18.67 & 3.78 \\
Other people support & 18.32 & 3.56 \\
Total score & 56.23 & 11.46 \\
\hline
\end{tabular}

virtual channels of the nurses and they were asked to complete the questionnaires if wish to participate in this study, they are working in the wards where COVID-19 patients were hospitalized. After the questionnaires were completed, their information was extracted and analysed using SPSS and PLS software.

Structural equation modelling method and PLS software were used to test the proposed model and existing hypotheses. There are two stages in the structural equation modelling to examine the model, including the measurement and structural model test. In PLS modelling, the measurement model is called the outer model and the structural model is called the inner model. The measurement model examines the validity and reliability of measurement tools and research structures and tests the structural model of hypotheses and relationships between latent variables [26].

\section{Results}

This study was conducted on 336 nurses working in the wards where COVID-19 patients were hospitalized with mean age $(36.41 \pm 9.24)$ and work experience (12.41 \pm 8.12$)$.

Table 1 shows the average workload score. As shown in this table, the highest and lowest scores belong to the dimension of temporal demand and performance, respectively. Table 2 shows the mean score of the perceived social support. As shown in this table, the highest score belongs to family support
Table 3

Mean quality of life score

\begin{tabular}{lcc}
\hline Quality of life & Mean & SD \\
\hline Physical health & 62.74 & 15.34 \\
Mental health & 54.78 & 12.48 \\
Social relationships & 47.36 & 12.63 \\
Environmental health & 58.63 & 14.52 \\
Total Score & 55.87 & 13.74 \\
\hline
\end{tabular}

Table 4

Measurement model results

\begin{tabular}{lcccc}
\hline Variables & $\begin{array}{c}\text { Factor } \\
\text { load }\end{array}$ & $\begin{array}{c}\text { Statistics } \\
\text { T }\end{array}$ & $\begin{array}{c}\text { Composite } \\
\text { reliability }\end{array}$ & AVE \\
\hline Work load & & & 0.764 & 0.609 \\
Mental demand & 0.738 & 13.276 & & \\
Physical demand & 0.686 & 10.643 & & \\
Temporal demand & 0.840 & 16.753 & & \\
Performance & 0.593 & 7.534 & & \\
Effort & 0.644 & 8.866 & & \\
Frustration & 0.670 & 9.475 & & \\
Perceived social support & & & 0.815 & 0.648 \\
Family support & 0.745 & 13.694 & & \\
Friends support & 0.687 & 10.834 & & \\
Other people support & 0.654 & 9.358 & & \\
Quality of life & & & 0.793 & 0.642 \\
Physical health & 0.746 & 14.017 & & \\
Mental health & 0.683 & 8.478 & & \\
Social relationships & 0.720 & 10.608 & & \\
Environmental health & 0.748 & 11.926 & & \\
\hline
\end{tabular}

and the lowest score depends on the support of others Table 3 shows the mean score of quality of life and its dimensions. As shown in this table, the highest and lowest scores belong to the physical and social dimensions, respectively. The assumed conceptual model implemented in PLS software is shown in Fig. 1. This figure includes a measurement and structural model. As shown in Fig. 2, workload has an adverse effect on quality of life (Coefficient $=-0.283$ ), but perceived social support has a direct effect on quality of life (Coefficient $=0.574)$. Perceived social support also has a moderating effect $($ Coefficient $=0.164)$ in the relationship between workload and quality of life and leads to reduce the effect of workload and reduce the quality of life.

The criteria of factor loading, T-statistic, composite reliability and average variance extracted (AVE) were used to evaluate the measurement model, the results of which are shown in Table 4. The measurement model has a good validity due to the appropriate values for the desired criteria (Loading $>0.4$, T satatistic $>1.95$, Composite reliability $>0.7, \operatorname{AVE}>0.5$ ) [26], and the values presented in Table 4 . T and $P$-value statistics were used to examine the structural model 


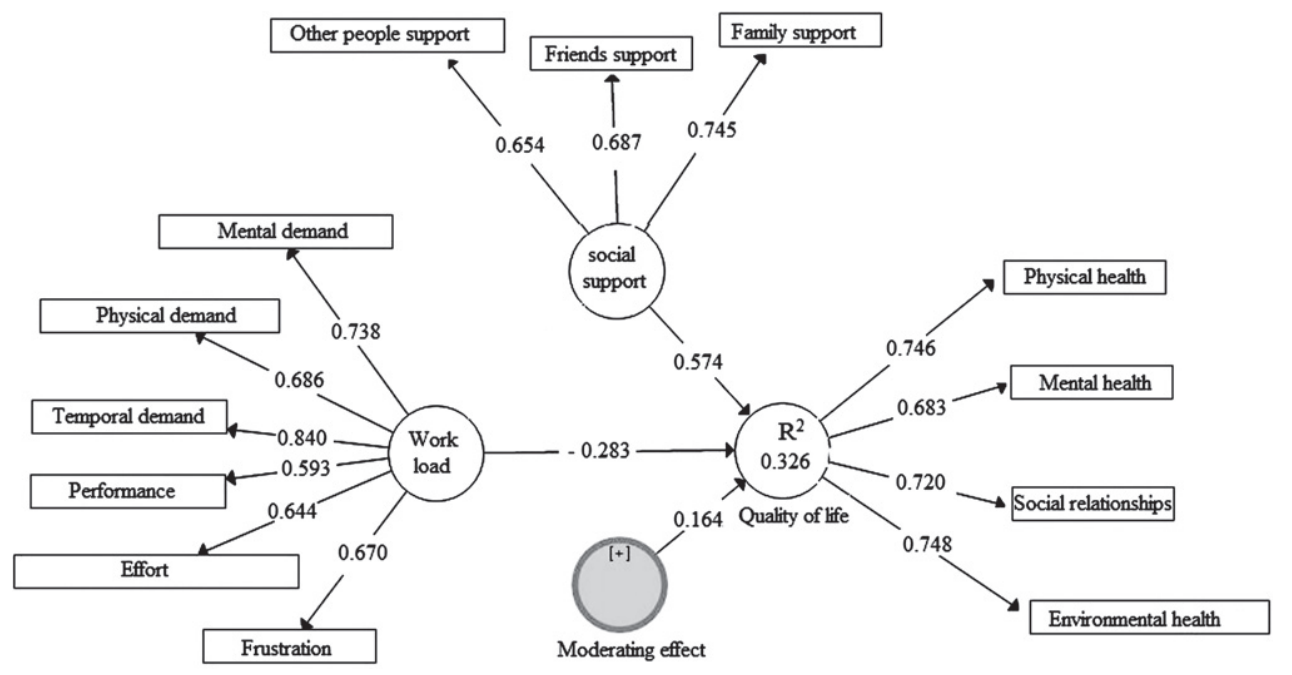

Fig. 2. The diagram of implemented structural equation model.

Table 5

Structural model results

\begin{tabular}{lccc}
\hline Factor relationships & Coefficient & T statistics & $P$-value \\
\hline Workload $\longrightarrow$ Quality of life & -0.283 & 4.648 & 0.008 \\
Perceived social support $\longrightarrow$ Quality of life & 0.574 & 7.015 & 0.001 \\
Moderating effect $\longrightarrow$ Quality of life & 0.164 & 2.034 & 0.041 \\
\hline
\end{tabular}

of coefficient criteria [26], the results of which are presented in Table 5. According to Table 5, workload has an inverse and significant effect on quality of life, but the perceived social support has a direct effect and significant on quality of life. Also, perceived social support by the moderating effect reduces the effect of workload on quality of life.

\section{Discussion}

According to the results of this study, the mean workload score in the nurses under study was high $(80.87 \pm 20.17)$ and the highest and lowest scores belong to the dimensions of time need, frustration demand, physical, mental, effort and performance, respectively. Time demand means the feeling pressured to do something on time, and the level of frustration means the amount of insecurity, discouragement, irritability, stress, and disturbance in the face of security, feeling of satisfaction, comfort and self-satisfaction during performing the task [27].

According to the results of studies, the duties of nurses are mental and information processing and rapid decision making is needed, and the highest score belongs to the mental dimension $[28,29]$. The results of this study showed that mental demand is placed after the time demand, frustration and physical dimensions which are inconsistent with the results of previous studies. In interpreting the results, it can be concluded that the COVID-19 pandemic has led to increase the number of patients and consequently increase the time pressure to perform tasks. Also, the lack of proper treatment for patients with COVID19 leads to death of some patients. Lack of proper treatment and death of patients can lead to increased stress and discouragement and irritability in nurses and finally the level of frustration in them will be increased. Also, the higher score of the physical dimension (the amount of physical activity required to perform tasks) compared to the mental dimension indicates an increase in the number of patients and an increase in the amount of physical work. The special conditions governing the hospital in the COVID-19 pandemic can be mentioned in this regard, so that nurses are forced to use special personal protective equipment such as protective clothes (gowns) and masks. The use of personal protective equipment can increase energy consumption and increase the physical dimension score. So, it can be concluded that the COVID-19 pandemic has led to increase the number of patients and increase physical workload, time pressure and level of frustration in nurses. 
According to the results of the Perceived Social Support Survey, the mean score of perceived social support in the nurses under study is relatively high and the highest score belongs to family support and the lowest score depends on the support of others. Based on the results reported by Eman et al, perceived social support for all dimensions was moderate-to-high in Jordanian healthcare workers [30]. The results of a study by Han et al showed the high level of perceived social support for medical staff during COVID-19 pandemic [31]. During the COVID-19 pandemic era, the activity of nurses working in the COVID-19 patients-related wards was out of the ordinary, and nurses were forced to stay out of contact with their family and friends for long periods of time and be in quarantine to prevent disease transmission. According to most nurses under study, the family understands their situation and encourages them to continue their work. Also, they have tried to maintain communication with nurses through virtually and to inspire them. For this reason, the family dimension score has been higher than the other dimensions. Also, the support dimension has received the lowest score, due to non-compliance with prevention protocols by some people in the community that leads to the spread of the disease.

According to the results of quality of life study, the mean score of quality of life in the nurses under study was relatively low and the highest score belongs to the physical, environmental, psychological and social dimensions, respectively. According to the results reported by Azizi et al., the mean score of quality of life in nurses was $55.92 \pm 12.2$ that the highest score of quality of life in nurses belongs to the physical, psychological, social and environmental dimensions, respectively [32]. According to the results reported by Bahrami, nurses do not have a good quality of life and the highest score belongs to the physical, social, psychological and environmental dimensions, respectively [33]. According to the results of comparison between studies conducted in Iran, the quality of life in Iranian nurses is moderate and low [33, 34]. The results of this study show that, the lowest score belonged to the social dimension. The social dimension of quality of life means the distortion of private and social relations Nurses working in the COVID-19 patients-related wards are forced to reduce their relationships with family and friends due to the COVID-19 pandemic and its high prevalence. Impossibility of communication with family and friends effectively can lead to decrease the quality of the social dimension of quality of life. The mental dimension of quality of life, which had the lowest score after the social dimension, means enjoying life, feeling that life is meaningful, power of concentration, satisfaction with one's mental image, self-satisfaction, as well as nurses' emotional, mood and depression state [33]. Feelings of depression, decreased performance satisfaction, decreased concentration and mood of nurses are results of lack of proper treatment for patients with COVID-19 and the death of some patients despite the efforts of medical staff. Also, the impossibility of communication with family effectively, in addition to reducing the social dimension, can lead to decrease the enjoyment of life and feeling that life is meaningful, and consequently reduce the mental dimension of quality of life. Environmental issues raise questions in relation with cases such as a sense of security, financial ability financial capacity, access to required information, recreational activities, access to health resources, with accommodation, and access to transportation facilities [33].

It seems that the outbreak of the COVID-19 pandemic has led to increase staff concerns about financial matters, and reduce job security and leisure. Because, according to the results of Bahrami, nurses are more concerned in this field [33]. On the other hand, the high score of this dimension of quality of life can be attributed to the organizational support provided in the COVID-19 pandemic to provide a suitable work environment for medical staff.

According to the results of the structural equation measurement modelling, the studied structures have high validity. The results of the structural model confirmed the hypotheses of the structural equation model. According to the results, workload has an inverse and significant effect on quality of life, but perceived social support has a direct and significant effect on quality of life. Also, perceived social support by the moderating effect reduces the effect of workload on quality of life.

Quality of life is a complex set of people's reactions to psychological, physical, and social factors that affect their natural life [35]. Quality of life means the ability of a person to do life-related activities satisfactorily [36]. Heavy workload can disrupt the work-life balance and the person may not be able to do the life-related activities and the quality of life may decrease. According to the results of this study, the COVID-19 pandemic has increased workload and consequently reduced the quality of life of nurses. Perceived social support can act a moderator under these circumstances and reduce the effect of workload on quality of life. Social support is a social network 
that provides considerable psychological resources for a person, so that he/she will be able to cope with stressful living conditions and daily problems [37]. Social support as one of the emotional coping methods can protect people by preventing the occurrence of stressful situations and traumatic events or enable them to evaluate stressful events as less threatening [38]. So, in interpreting the results, it can be said that workload of nurses has increased in the COVID-19 pandemic era, and perceived social support reduces the negative effect of workload on quality of life by moderating stress and increasing individual performance.

\section{Limitations}

This study is not without limitaitons. We can point to the lack of attention to stress as a mediator between workload and quality of life, the small sample size, and a large number of confounding variables.

\section{Conclusion}

According to the results of the study, the COVID19 pandemic has led to an increased workload and has decreased the quality of life of nurses. The results showed that workload has an inverse and significant effect on quality of life, but perceived social support has a direct and significant effect on quality of life. Also, perceived social support by the moderating effect reduces the effect of workload on quality of life. Heavy workload can disrupt the balance between work and life and reduce a person's inability to do things in life satisfactorily and lead to a reduced quality of life. Meanwhile, perceived social support can reduce the negative effect of workload on quality of life through increasing performance. Therefore, it is recommended that the level of social support for nurses is increased to support the medical staff during the COVID-19 pandemic era, so that social support as a moderator can reduce the negative effects of workload.

\section{Acknowledgments}

The authors would like to thank all nurses who participated in the study.

\section{Conflict of Interest}

The authors declare that there is no conflict of interest regarding the publication of this article.

\section{References}

[1] Zhu H, Wei L, Niu P. The novel coronavirus outbreak in Wuhan, China. Global Health Research and Policy. 2020; 5(1):1-3.

[2] Zandian H, Sarailoo M, Dargahi S, Gholizadeh H, Vosoughi M, Dargahi A. Evaluation of knowledge and health behavior of university of medical sciences students about the prevention of COVID-19. Work. 2021;1(Preprint):1-7.

[3] Zhao S, Chen H. Modeling the epidemic dynamics and control of COVID-19 outbreak in China. Quantitative Biology. 2020:1-9.

[4] Liu X, Na R, Bi Z. Challenges to prevent and control the outbreak of Novel Coronavirus Pneumonia (COVID-19). Zhonghua liu xing bing xue za zhi=Zhonghua liuxingbingxue zazhi. 2020;41:E029-E029.

[5] Lai C-C, Shih T-P, Ko W-C, et al. Severe acute respiratory syndrome coronavirus 2 (SARS-CoV-2) and corona virus disease-2019 (COVID-19): the epidemic and the challenges. International journal of antimicrobial agents. 2020:105924.

[6] de Jong AE, Leeman J, Middelkoop E. Development of a nursing workload measurement instrument in burn care. Burns. 2009;35(7):942-948.

[7] Myny D, Van Hecke A, De Bacquer D, Verhaeghe S, Gobert M, Defloor T, Van Goubergen D. Determining a set of measurable and relevant factors affecting nursing workload in the acute care hospital setting: a cross-sectional study. International journal of nursing studies. 2012;49(4):427-36.

[8] Pennbrant S, Dåderman A. Job demands, work engagement and job turnover intentions among registered nurses: Explained by work-family private life inference. Work. 2021;10(Preprint):1-3.

[9] Arslan SS, Alemdaroğlu İ, Karaduman AA, Yilmaz ÖT. The effects of physical activity on sleep quality, job satisfaction, and quality of life in office workers. Work. 2019;63(1):3-7.

[10] Queijo AF, Martins RS, Andolhe R, Oliveira EM, Barbosa RL, Padilha KG. Nursing workload in neurological intensive care units: cross-sectional study. Intensive and Critical Care Nursing. 2013;29(2):112-6.

[11] Hazavehei SMM, Hamidi Y, Kharghani Moghadam SM, et al. Exploring the views of medical staff in transforming a hospital into a health promoting hospital in Iran: a qualitative research. Hospital Practice. 2019;47(5):241-248.

[12] Mohammadpour RA. Health-related quality of life of a general population over 40 years old in Mazandaran province, Iran. Payesh (Health Monitor). 2011;10(2):145-149.

[13] Gil-Lacruz M, Gil-Lacruz AI, Gracia-Pérez ML. Healthrelated quality of life in young people: the importance of education. Health and Quality of Life Outcomes. 2020;18(1):1-3.

[14] Haraldstad K, Wahl A, Andenæs R, Andersen JR, Andersen $\mathrm{MH}$, Beisland E, Borge CR, Engebretsen E, Eisemann M, Halvorsrud L, Hanssen TA. A systematic review of quality of life research in medicine and health sciences. Quality of life Research. 2019;28(10):2641-50.

[15] Pett MA, Guo JW, Cardell B, Johnson EP, Guerra N, Clark L. Psychometric properties of a brief self-reported 
health-related quality of life measure (HRQoL-IDD) for persons with intellectual and developmental disabilities. Journal of Applied Research in Intellectual Disabilities. 2021;34(3):877-90.

[16] Md-Sidin S, Sambasivan M, Ismail I. Relationship between work-family conflict and quality of life: An investigation into the role of social support. Journal of Managerial Psychology. 2010;26.

[17] Lin X, Kishore R. Social media-enabled healthcare: a conceptual model of social media affordances, online social support, and health behaviors and outcomes. Technological Forecasting and Social Change. 2021;166:120574.

[18] Schwartz A, Gerberich SG, Albin T, Kim H, Ryan AD, Church TR, Green DR, McGovern PM, Erdman AG, Arauz $R$. The association between janitor physical workload, mental workload, and stress: The SWEEP study. Work. 2020; 15(Preprint):1-0.

[19] Malekpour F, Fazli Ochhesar B, Mohammadpour Y, et al. Assessmen of relationship between quality of life and mental workload among nurses of Urmia Medical Science University hospitals. The Journal of Urmia Nursing and Midwifery Faculty. 2014;12(6):499-505.

[20] Zakerian SA, Abbasinia M, Mohammadian F, et al. The relationship between workload and quality of life among hospital staffs. Iranian Journal of Ergonomics. 2013;1(1): 43-56.

[21] Gurses AP, Carayon P, Wall M. Impact of performance obstacles on intensive care nurses' workload, perceived quality and safety of care, and quality of working life. Health Services Research. 2009;44(2p1):422-43.

[22] Brown TA, Moore MT. Confirmatory factor analysis. Handbook of Structural Equation Modeling. 2012:361-79.

[23] Nejat S, Montazeri A, Holakouie Naieni K, et al. The World Health Organization quality of Life (WHOQOL-BREF) questionnaire: Translation and validation study of the Iranian version. Journal of School of Public Health and Institute of Public Health Research. 2006;4(4):1-12.

[24] World Health Organization. WHOQOL-BREF: introduction, administration, scoring and generic version of the assessment: field trial version, December 1996. World Health Organization; 1996.

[25] Hosseini A, Mjdy A, Hassani G. Investigating the role of social support on the quality of life of the elderly in Mashhad in 2014. Journal of Gerontology. 2016;1(2):10-8.

[26] Hair JF, Ringle CM, Sarstedt M. PLS-SEM: Indeed a silver bullet. Journal of Marketing theory and Practice. 2011; 19(2):139-52.
[27] Marras WS, Karwowski W. Fundamentals and assessment tools for occupational ergonomics. Crc Press; 2006.

[28] Arghami S, Kamali K, Radanfar F. Task performance induced work load in nursing. Journal of Occupational Hygiene Engineering. 2015;2(3):45-54.

[29] Hoonakker P, Carayon P, Gurses AP, et al. Measuring workload of ICU nurses with a questionnaire survey: the NASA Task Load Index (TLX). IIE Transactions on Healthcare Systems Engineering. 2011;1(2):131-43.

[30] Alnazly E, Khraisat OM, Al-Bashaireh AM, Bryant CL. Anxiety, depression, stress, fear and social support during COVID-19 pandemic among Jordanian healthcare workers. Plos one. 2021;16(3):e0247679.

[31] Xiao H, Zhang Y, Kong D, Li S, Yang N. The effects of social support on sleep quality of medical staff treating patients with coronavirus disease 2019 (COVID-19) in January and February 2020 in China. Medical science monitor: international medical journal of experimental and clinical research. 2020;26:e923549-1.

[32] Azizi M, BAROONY ZZ, Motamedzade M, et al. Study of nurses quality of life using WHO questionnaire in hospitals of Hamadan University of Medical Sciences. 2015.

[33] Bahrami M. Nurses' quality of life in medical-surgical wards of an oncology center affiliated to the Isfahan University of Medical Sciences. Nursing Journal of the vulnerable. 2016;3(7):36-46.

[34] Hamidi Y, Hazavehei SMM, Karimi-Shahanjarini A, et al. Health promoting hospitals in Iran: A review of the current status, challenges, and future prospects. Medical journal of the Islamic Republic of Iran. 2019;33:47.

[35] Pais-Ribeiro J. Quality of life is a primary end-point in clinical settings. Clinical nutrition. 2004;23(1):121-30.

[36] Jones CJ, Rose DJ. Physical activity instruction of older adults. Human Kinetics; 2005.

[37] Cohen S, McKay G. Social support, stress and the buffering hypothesis: A theoretical analysis. InHandbook of Psychology and Health (Volume IV) 2020;10(pp. 253-267). Routledge.

[38] Roberts ME, Bernstein MH, Colby SM. The effects of eliciting implicit versus explicit social support among youths susceptible for late-onset smoking. Addictive Behaviors. 2016;62:60-4. 